Pesq. Vet. Bras. 34(Supl.1):39-42, dezembro 2014 DOI: 10.1590/S0100-736X2014001300008

\title{
Determinação das concentrações plasmáticas de proteínas e metabólitos de cascavéis em cativeiro ${ }^{1}$
}

\author{
Danielle S. Vieira ${ }^{2 *}$, Thaís C.S. Rodrigues ${ }^{3}$, Renata L. de Miranda ${ }^{3}$, \\ Graciele F. Cardoso ${ }^{4}$, Ednaldo C. Guimarães ${ }^{3}$, Vera L.C. Brites ${ }^{4}$ e Antonio V. Mundim ${ }^{3}$
}

\begin{abstract}
Vieira D.S., Rodrigues T.C.S., de Miranda R.L., Cardoso G.F., Guimarães E.C., Brites V.L.C. \& Mundim A.V. 2014. [Determination of proteins and metabolites concentrations in plasma of rattlesnakes in captivity.] Determinação das concentrações plasmáticas de proteínas e metabólitos de cascavéis em cativeiro. Pesquisa Veterinária Brasileira 34(Supl.1):39-42. Laboratório Clínico Veterinário, Faculdade de Medicina Veterinária, Universidade Federal de Uberlândia, Av. Mato Grosso 3289, Uberlândia, MG 38405-314, Brasil. E-mail: danivbio@gmail.com

For determining plasma concentrations of proteins and metabolites of rattlesnakes in captivity, 60 adult snakes, 30 males and 30 females were used. Blood was collected by puncture of the cervical paravertebral venous sinus and stored in tubes with heparin. Biochemical analyzes were colorimetrically processed using an Automatic Biochemistry Analyzer Chemwell (Awareness Technology ${ }^{\circledR}$, Inc). The mean and standard deviation were calculated for the following constituents: total protein, albumin, globulin, albumin/globulin ratio, uric acid, creatinine, urea, cholesterol, HDL cholesterol and triglycerides. The values were similar to those previously reported for reptiles and snakes, with the differences observed probably due to the difference between species, climate, season and the methodology used. There were no significant differences between males and females for the parameters studied. These results may be useful in establishing normal biochemical values for conservation plans for these snakes in captivity.
\end{abstract}

INDEX TERMS: Blood biochemistry, Crotalus durissus, reptile, Viperidae, rattle snake.

RESUMO.- Para determinar as concentrações plasmáticas de proteínas e metabólitos de cascavéis em cativeiro, foram utilizadas 60 serpentes adultas, sendo 30 machos e 30 fêmeas. 0 sangue foi coletado através de punção do seio venoso paravertebral cervical e armazenado em tubos com heparina. As análises bioquímicas foram processadas colorimetricamente em Analisador Automático de Bioquímica Chemwell (Awareness Technology ${ }^{\circledR}$, Inc). Foram calculadas as médias e desvios padrão dos seguintes cons-

\footnotetext{
${ }^{1}$ Recebido em 11 de julho de 2014.

Aceito para publicação em 13 de dezembro de 2014.

${ }^{2}$ Laboratório de Patologia Clínica Veterinária, Faculdade de Medicina Veterinária, Universidade Federal de Uberlândia. (UFU), Av. Mato Grosso 3289, Bloco 2S, Campus Umuarama, Uberlândia, MG 38405-314, Brasil. *Autor para correspondência: danivbio@gmail.com

${ }^{3}$ Laboratório de Patologia Clínica Veterinária, Faculdade de Medicina Veterinária, UFU, Av. Mato Grosso 3289, Bloco 2S, Campus Umuarama, Uberlândia, MG 38405-314.

${ }^{4}$ Laboratório de Herpetologia, Setor de Manutenção de Répteis, Instituto de Biologia, UFU, Rua Ceará, s/n, Bloco 2D, Campus Umuarama, Uberlândia, MG 38400-902.
}

tituintes: proteínas totais, albumina, globulinas, relação albumina/globulinas, ácido úrico, creatinina, ureia, colesterol, colesterol HDL e triglicérides. Os valores obtidos foram semelhantes aos descritos na literatura para repteis e serpentes, sendo as diferenças observadas provavelmente decorrentes da diferença entre espécies, clima, estação do ano e metodologia utilizada. Não houve diferenças significativas entre machos e fêmeas para os parâmetros estudados. Estes resultados podem ser úteis no estabelecimento de valores de referência para planos de conservação destes ofídios em cativeiro.

TERMOS DE INDEXAÇÃO: Bioquímica sanguínea, cascavel, Crotalus durissus, réptil, Viperidae, serpente.

\section{INTRODUÇÃO}

A cascavel (Crotalus durissus Linnaeus, 1758) é uma serpente da família Viperidae, com ampla distribuição desde o México até a Argentina, e a única representante do gênero Crotalus no Brasil (Campbell \& Lamar 1989). São facilmente identificadas pela presença de um guizo ou chocalho na 
extremidade da cauda, que é vibrado quando a serpente se sente ameaçada, emitindo um som que afugenta os predadores (Pinho, Vidal \& Burdmann 2000).

Tem se tornado frequente o aparecimento de serpentes em áreas urbanas, devido ao acelerado crescimento das cidades e da população humana, que causa aumento do desmatamento e da fragmentação das áreas naturais. Como consequência, houve aumento no número de casos de acidentes ofídicos, sendo as cascavéis responsáveis por apenas $7,7 \%$ desses casos, porém, com a maior taxa de letalidade (Araújo, Santalúcia \& Cabral 2003). Em função disso, as serpentes são criadas em cativeiro por institutos de pesquisa para obtenção de peçonha para produção de soro antiofídico e estudo de seus constituintes (Serapicos \& Merusse 2002).

Os constituintes do sangue refletem com precisão o estado metabólico do organismo. Porém, a interpretação dos resultados bioquímicos nem sempre é clara, pois podem sofrer interferências de fatores como sexo, idade, sazonalidade, estado nutricional, entre outros, principalmente em animais ectotérmicos (Campbell 2006). 0 exame físico em animais silvestre, especialmente nos répteis, é difícil, sobretudo em se tratando de animais peçonhentos como serpentes. Por isso, o estudo da bioquímica sanguínea é importante para o conhecimento do estado de saúde, diagnóstico, prognóstico e tratamento de doenças em medicina veterinária.

As proteínas são um grupo de constituintes do sangue importantes na manutenção da pressão oncótica, tamponamento de alterações do pH, imunidade humoral, atividade enzimática, coagulação e resposta de fase aguda. Os metabólitos são os intermediários e os produtos do metabolismo, como o colesterol, triglicérides, creatinina, ureia, entre outros. A concentração sanguínea alterada desses metabólitos pode indicar desequilíbrio nutricional ou alguma alteração orgânica na capacidade de utilização ou biotransformação de nutrientes (Wittwer 1995).

0 estudo do perfil bioquímico de cascavéis pode fornecer informações importantes sobre as condições metabólicas gerais destes animais, auxiliando na manutenção das populações em cativeiro e no diagnostico de várias enfermidades.

Objetivou-se com este estudo determinar os valores de proteínas e metabólitos do plasma de cascavéis mantidas em cativeiro e verificar se há diferença entre machos e fêmeas.

\section{MATERIAL E MÉTODOS}

Foram utilizadas 60 serpentes adultas da subespécie $C$. $d$. collilineatus, sendo 30 machos e 30 fêmeas, clinicamente saudáveis, mantidas em cativeiro no Setor de Repteis do Instituto de Biologia da Universidade Federal de Uberlândia.

As serpentes foram contidas manualmente por técnicos treinados, utilizando ganchos, como indicado por Francisco (1997) e Goulart (2004). Após a contenção, as presas solenóglifas foram fixadas a pedaços de isopor com o uso de pinças (Fig.1A), de maneira a impedir que o animal pudesse se soltar e permitindo livre acesso à cabeça da serpente. A colheita do sangue $(2 \mathrm{~mL})$ foi realizada após prévia assepsia com álcool 70\%, por punção do seio venoso paravertebral cervical, descrito por Zippel, Lillywhite e Mladinich (2001). A punção foi feita entre o osso occipital e o atlas, com agulha $0,45 \times 13 \mathrm{~mm}$ e seringa de $5 \mathrm{~mL}$ (Fig.1B). As amostras foram armazenadas em tubos de $3 \mathrm{~mL}$ contendo heparina, e posteriormente centrifugadas a $720 \mathrm{~g}$ durante cinco minutos em centrifuga Baby 2-Fanem ${ }^{\circledR}$ para a obtenção do plasma. As análises bioquímicas foram processadas colorimetricamente em Analisador Automático de Bioquímica Chemwell (Awareness Technology ${ }^{\circledR}$, Inc), previamente calibrado com calibra $\mathrm{H}$ e aferido com soro controle qualitrol $\mathrm{H}$.

Os parâmetros bioquímicos analisados foram: proteína total (método biureto), albumina (método verde de bromocresol), globulinas (cálculo: proteína total - albumina), relação albumina/ globulinas (A/G) (cálculo: albumina/globulinas), ácido úrico (método enzimático Trinder), creatinina (método picrato alcalino), ureia (método enzimático UV), colesterol (método enzimático Trinder), triglicérides (método enzimático Trinder) e lipoproteínas de alta densidade transportadoras de colesterol HDL-C (método Labtest), utilizando kits da Labtest Diagnóstica ${ }^{\circledR}$.

Para verificar se houve diferença significativa entre machos e fêmeas foi utilizado o teste t para amostras independentes quando se verificou normalidade e teste de Wilcoxon-Mann-Whitney quando não foi observada a distribuição normal. 0 teste de normalidade aplicado foi o teste de Anderson-Darling com signifi-

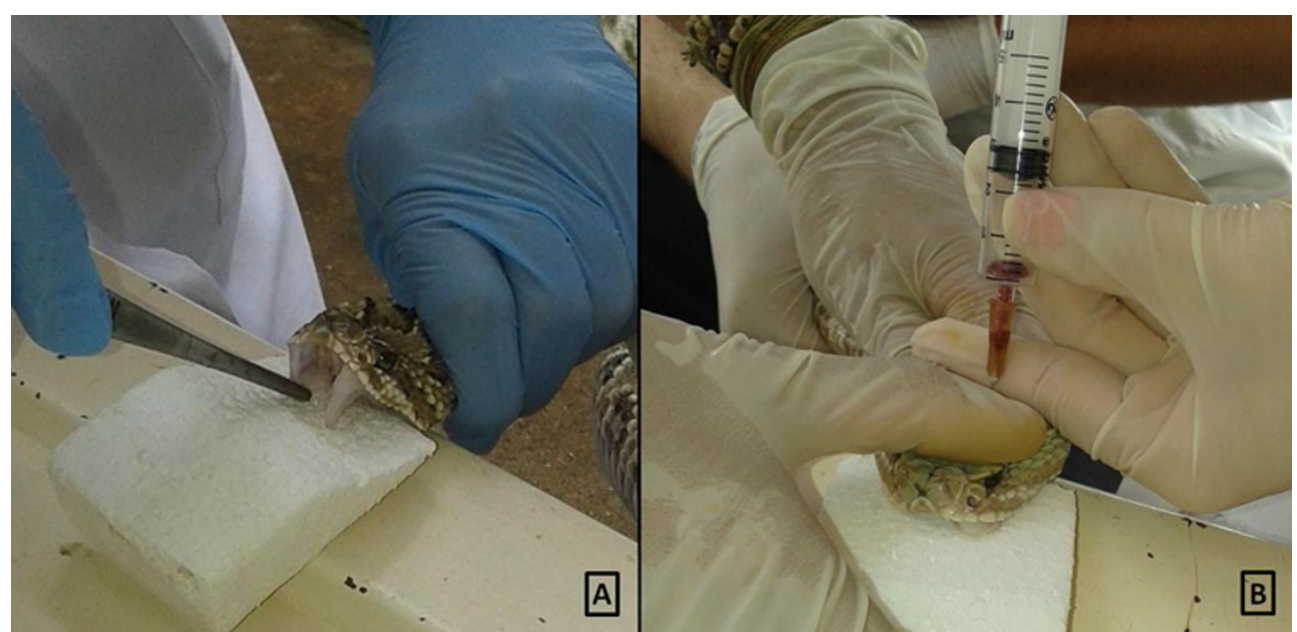

Fig.1. (a) Fixação das presas solenóglifas a pedaços de isopor; (b) Punção do seio venoso paravertebral cervical. 
cância de 5\%. Os procedimentos de análises estatísticas foram feitos na ferramenta Action que utiliza o programa R (R Development Core Team). A pesquisa teve aprovação da Comissão de Ética na Utilização de Animais (CEUA) da Universidade Federal de Uberlândia, conforme protocolo no $149 / 13$, e do SISBIO número 42135-1.

\section{RESULTADOS E DISCUSSÃO}

Os resultados dos parâmetros bioquímicos plasmáticos mensurados encontram-se no quadro 1 . 0 valor de proteína total obtido no presente estudo foi maior que o encontrado por Troiano et al. (2001) e Silva et al. (2010) para o mesmo gênero $(3,79 \pm 0,41 \mathrm{~g} / \mathrm{dL}$ e $3,7 \pm 0,7 \mathrm{~g} / \mathrm{dL}$ respectivamente), e também maior que valores encontrados para o gênero Bothrops (B. jararaca: 3,34 $\pm 1,07 \mathrm{~g} / \mathrm{dL}$ e B. jararacussu 2,94 $\pm 1,31 \mathrm{~g} / \mathrm{dL}$ ) (Glaser et al. 2013) (B. ammodytoides 4,08 $\pm 0,28 \mathrm{~g} / \mathrm{dL}$ ) (Troiano et al. 1999), mas está dentro dos valores descrito para repteis por Campbell (2006), entre 3,0 e $7,0 \mathrm{~g} / \mathrm{dL}$.

0 valor de albumina foi semelhante aos encontrados por Troiano et al. (2001) e Silva et al. (2010) $(1,77 \pm 0,31 \mathrm{~g} /$ $\mathrm{dL}, 1,62 \pm 0,4 \mathrm{~g} / \mathrm{dL}$ respectivamente), e as globulinas foram maiores que os valores encontrados por esses autores (Troiano et al. 2001: 2,03 $\pm 0,29 \mathrm{~g} / \mathrm{dL}$ e Silva et al.2010: $2,08 \pm 0,50 \mathrm{~g} / \mathrm{dL}$ ). Em répteis saudáveis a albumina é a maior fração proteica. Em condições inflamatórias pode ocorrer aumento da proteína total causada pela elevação da fração de globulinas, e algumas doenças podem causar decréscimo na relação albumina/globulinas (A/G). Altas concentrações de proteína com relação $\mathrm{A} / \mathrm{G}$ normal indicam desidratação (Knotek et al. 2011). Caso a hiperproteinemia esteja associada com hipoalbuminemia e hiperglobulinemia pode indicar doença inflamatória crônica. Neste estudo foram observados valores mais elevados de proteína total, sendo a fração globulinas maior que a albumina, apesar dos animais estarem clinicamente sadios. Hipoproteinemia e hipoalbuminemia em répteis estão associadas à má nutrição ou doença hepática e renal severa (Almosny \& Monteiro 2007).

Os valores de ácido úrico, creatinina e ureia estão de acordo com o descrito por Campbell (2006) para repteis terrestres $(<10 \mathrm{mg} / \mathrm{dL},<1,0 \mathrm{mg} / \mathrm{dL}$ e $<15 \mathrm{mg} / \mathrm{dL}$, respectivamente), e são semelhantes aos encontrados por Troiano et al. (2001), Silva et al. (2010) e Glaser et al. (2013), porem, estes parâmetros não são confiáveis para avaliar a função renal destes animais. 0 ácido úrico é o principal produto final do metabolismo do nitrogênio em répteis terrestres, excretado em forma pouco solúvel e semi-sólida, sendo por isso considerado uricotélicos (Campbell 2006). A hiperuricemia pode estar associada a dietas ricas em proteínas, desidratação e gota. A concentração de ácido úrico nos répteis também pode aumentar em casos de desnutrição grave, devido ao aumento do catabolismo de compostos nitrogenados. Répteis carnívoros tem aumento de até duas vezes nas concentrações de ácido úrico após a alimentação. A dosagem de ácido úrico não é indicada para diagnosticar doença renal crônica nesses animais, porque é necessária perda de aproximadamente $75 \%$ da função renal para aumentar suas concentrações sanguíneas (Knotek et al. 2011). As concentrações de ureia e creatinina em répteis são baixas, devido ao fato de serem uricotélicos, indicando apenas o estado de hidratação (Campbell, 2006).

Os valores de colesterol e triglicérides encontrados nesse estudo concordam com os encontrados por Silva et al. (2010), mas Troiano et al. (2001) encontraram valores menores de colesterol e maiores de triglicérides. Em outros viperídeos, como B. ammodytoides estudados por Troiano et al. (1999), os valores foram semelhantes. B. alternatus teve valores menores de colesterol e semelhantes de triglicérides (Troiano et al. 1998). Zierer et al. (2008) mensuraram os valores de colesterol, triglicérides e HDL em espécimes de $B$. jararaca em cativeiro e vida livre. Os valores de triglicérides e colesterol obtidos por Zierer et al. (2008) para animais de cativeiro e vida livre concordaram com os do presente estudo. 0 valor de HDL-C concorda com o de animais em cativeiro $(35,6 \pm 9,29 \mathrm{mg} / \mathrm{dL})$ e foi menor que o encontrado para espécimes de vida livre $(243,36 \pm 113,27 \mathrm{mg} /$ dL). Esta lipoproteína apresenta valores mais baixos para animais sedentários. Valores elevados de colesterol e triglicérides podem estar relacionados com doença hepática grave ou estro em fêmeas (Dessauer 1970).

A comparação dos valores entre machos e fêmeas encontra-se no quadro 2. Não houve diferença significativa entre machos e fêmeas para os parâmetros analisados. De acordo com Campbell (2006), dentro do período reprodutivo (fevereiro-março, novembro-janeiro) as concentrações plasmáticas de cálcio, colesterol e triglicérides são signifi-

Quadro 1. Valores médios, desvios padrão, mínimo e máximo dos parâmetros bioquímicos plasmáticos das cascavéis

\begin{tabular}{lccc}
\hline Parâmetros bioquímicos & Média \pm Desvio & Mínimo & Máximo \\
\hline Albumina (g/dL) & $1,26 \pm 0,28$ & 0,50 & 1,90 \\
Proteína total (g/dL) & $5,70 \pm 0,94$ & 3,70 & 7,80 \\
Globulinas (g/dL) & $4,44 \pm 0,87$ & 2,50 & 6,50 \\
Relação albumina/globulinas (A/G) & $0,30 \pm 0,09$ & 0,15 & 0,54 \\
Ácido Úrico (mg/dL) & $1,39 \pm 0,94$ & 0 & 4,80 \\
Creatinina (mg/dL) & $0,48 \pm 0,21$ & 0,11 & 1,20 \\
Ureia (mg/dL) & $8,43 \pm 8,37$ & 0,30 & 58,60 \\
Colesterol (mg/dL) & $207,40 \pm 69,60$ & 34,20 & 430,50 \\
HDL-C (mg/dL)* & $33,53 \pm 16,29$ & 4,30 & 95,90 \\
Triglicérides (mg/dL) & $60,12 \pm 84,74$ & 1,40 & 451,60
\end{tabular}

* HDL-C = Lipoproteinas de alta densidade transportadoras de Colesterol.

Quadro 2. Valores médios e desvios padrão dos parâmetros bioquímicos plasmáticos de machos e fêmeas

\begin{tabular}{lcccc}
\hline Parâmetros bioquímicos & \multicolumn{1}{c}{ Machos } & & Fêmeas \\
\cline { 2 - 2 } & Média \pm Desvio & & Média \pm Desvio \\
\hline Albumina (g/dL) & $1,30 \pm 0,28^{\mathrm{a}}$ & & $1,23 \pm 0,27^{\mathrm{a}}$ \\
Proteína total (g/dL) & $5,86 \pm 1,01^{\mathrm{a}}$ & & $5,53 \pm 0,84^{\mathrm{a}}$ \\
Globulinas (g/dL) & $4,57 \pm 0,95^{\mathrm{a}}$ & & $4,31 \pm 0,79^{\mathrm{a}}$ \\
Relação albumina/globulinas (A/G) & $0,30 \pm 0,09^{\mathrm{a}}$ & & $0,30 \pm 0,09^{\mathrm{a}}$ \\
Ácido Úrico (mg/dL) & $1,31 \pm 0,94^{\mathrm{a}}$ & & $1,47 \pm 0,95^{\mathrm{a}}$ \\
Creatinina (mg/dL) & $0,52 \pm 0,26^{\mathrm{a}}$ & & $0,44 \pm 0,15^{\mathrm{a}}$ \\
Uréia (mg/dL) & $9,21 \pm 10,25^{\text {a* }}$ & & $7,64 \pm 6,01^{\text {a* }}$ \\
Colesterol (mg/dL) & $201,19 \pm 70,28^{\mathrm{a}}$ & $213,62 \pm 69,54^{\mathrm{a}}$ \\
HDL-C (mg/dL)** & $28,83 \pm 11,52^{\mathrm{a} *}$ & $38,23 \pm 19,01^{\text {a* }}$ \\
Triglicérides (mg/dL) & $49,36 \pm 53,32^{\text {a* }}$ & & $70,87 \pm 107,36^{\mathrm{a} *}$
\end{tabular}

$\overline{a, b}$ Letras diferentes nas linhas, indicam diferença significativa entre os sexos $(\mathrm{p}<0,05) .{ }^{*}$ Teste de Wilcoxon-Mann-Whitney, demais parâmetros teste $\mathrm{t}$ para amostras independentes. ${ }^{* *} \mathrm{HDL}-\mathrm{C}=$ Lipoproteinas de alta densidade transportadoras de Colesterol. 
cativamente maiores em iguanas verdes fêmeas do que em machos. No período de foliculogênese há uma hiperproteinemia por causa de aumento na fração globulinas induzido pelo estrógeno. Apesar de não ser estatisticamente significante, no presente estudo as fêmeas apresentaram valores ligeiramente superiores de colesterol e triglicérides.

\section{CONCLUSÕES}

Os valores dos parâmetros bioquímicos sanguíneos de cascavéis no presente estudo em geral são semelhantes aos descritos na literatura para repteis e serpentes, sendo as diferenças observadas provavelmente decorrentes da diferença entre espécies, clima, período do ano e metodologia utilizada.

Não houve diferenças significativas entre machos e fêmeas para os parâmetros estudados. Estes resultados podem ser úteis no estabelecimento de valores de referência para planos de conservação destes ofídios em cativeiro.

\section{REFERÊNCIAS}

Almosny N.R.P. \& Monteiro A.M. 2007. Patologia clínica, p.939-967. In: Cubas Z.S., Silva J.C.S. \& Catão-Dias J.L. (Eds), Tratado de Animais Selvagens: Medicina Veterinária. Roca, São Paulo.

Araújo F.A.A., Santalúcia M. \& Cabral R.F. 2003. Epidemiologia dos acidentes por animais peçonhentos, p.6-12. In: Cardoso J.L.C., França F.O.S., Wen F.H., Malaque C.M.S. \& Haddad Jr. V. (Eds), Animais Peçonhentos no Brasil: biologia, clínica e terapêutica dos acidentes. Sarvier, São Paulo.

Campbell J.A. \& Lamar W.W. 1989. The Venomous Reptiles of Latin America. Cornell University Press, Ithaca. 425p.

Campbell T.W. 2006. Clinical pathology of reptiles, p.490-532. In: Mader D.R. (Ed.), Reptile Medicine and Surgery. Saunders Elsevier, Missouri.

Dessauer H.C. 1970. Blood chemistry of reptiles, physiological and evolutionary aspects, p.1-72. In: Gans C. (Ed.), Biology of the Reptilia. Academic Press, Londres.

Francisco L.R. 1997. Répteis do Brasil: manutenção em cativeiro. Editora Amaro, São José dos Pinhais. 207p.

Glaser V., Boni A.P., Pitz H.S., Albuquerque C.A.C. \& Zeni A.L.B. 2013. Pa- râmetros hematológicos e bioquímicos de Bothropoides jararaca e Bothrops jararacussu (Ophidia-Viperidae) mantidas em cativeiro. Archs Vet. Sci. 18:68-74.

Goulart C.E.S. 2004. Herpetologia, Herpetocultura e Medicina de Répteis. L.F. Livros e Veterinária, Rio de Janeiro. 330p.

Knotek Z., Knotkova Z. \& Hrdá A. 2011. Clinical Haematology and Plasma Chemistry in Reptiles. ${ }^{\text {th }}$ ed. Southern European Veterinary Conference (SEVC) Sept. 20 to Oct. 2. Barcelona, Espanha.

Pinho F.O., Vidal E.C. \& Burdmann E.A. 2000. Atualização em insuficiência renal aguda: insuficiência renal aguda após acidente crotálico. J. Bras. Nefrol. 22:162-168.

Serapicos E.O. \& Merusse J.L.B. 2002. Variação de peso e sobrevida de $M i$ crurus corallinus sob diferentes condições de alimentação em biotério (serpentes, Elapidae). Iheringia, Sér. Zool., Porto Alegre, 92:105-109.

Silva W.B., Soares R.M., Machado C., Freire I.M.A., Silva L.C.C.P., Moreira S.B., Goldberg D.W. \& Almosny N.R.P. 2010. Bioquímica plasmática de cascavéis (Caudisona durissa Linnaeus, 1758) em cativeiro. Ciência Rural 40:2510-2514.

Troiano J.C., Gould E., Malinskas G., Vidal J.C., Scaglione M.C., Gould J., Dinápoli H., Scaglione L.M. \& De Roodt A. 1998. Valores de bioquímica sanguínea de Bothrops alternatus em cautividad. Revta Espanhol. Herpetologia 12:45-48.

Troiano J.C., Vidal J.C., Gould E.F., Malinskas G., Gould J., Scaglione M., Scaglione L., Heker J.J., Simoncini C. \& Dinápoli H. 1999. Haematological and blood chemical values from Bothrops ammodytoides in captivity. Comp. Haematol. Int. 9:31-35.

Troiano J.C., Gould E.G., Althaus R., Malinskas G., Gould J.A., Heker J., Vidal J.C., Amantini E. \& Simoncini C. 2001. Blood biochemical profile of the south american rattlesnakes (Crotalus durissus terrificus) in captivity. J. Venom. Anim. Toxins 7:183-189.

Wittwer F. 1995. Empleo de los perfiles metabólicos em el diagnóstico de desbalences metabólicos nutricionales em el ganado. Buiatria, Goiânia, 2:16-20.

Zierer M., Brandão C.A.L., Foeger V., Daleprane J.M. \& Waiandt H.R. 2008. Estudo bioquímico do sangue de Bothrops jararaca (serpentes, Viperidae) mantida em cativeiro e de vida livre nos municípios de Santa Teresa e Santa Maria de Jetibá, ES. Diálogos e Ciência, Revista da Rede de Ensino FTC, 6:1-8.

Zippel K.C., Lillywhite H.B. \& Mladinich C.R.J. 2001. New vascular system in reptiles: Anatomy and postural hemodynamics of the vertebral venous plexus in snakes. J. Morphol. 250:173-184. 